
internationales

vol. $32-n^{\circ} 1 \mid 2016$

Les migrations des Roms roumains en Europe

\title{
Roms roumanisés, Ciurari, Ursari : ethnicité et appartenances sociales. Ethnographie des migrants roms roumains à Montpellier
}

Romanized Roma, Ciurari, Ursari: Ethnicity and Social Belongings.

Ethnography of Romanian Roma Migrants in Montpellier

Roma romanizados, Ciurari, Ursari: etnicidad y pertenencias sociales.

Etnografía de los roma rumanos migrantes en Montpellier

\section{Marion Lièvre}

\section{(2) OpenEdition}

Édition électronique

URL : https://journals.openedition.org/remi/7625

DOI : 10.4000/remi.7625

ISSN : $1777-5418$

Éditeur

Université de Poitiers

Édition imprimée

Date de publication : 1 mars 2016

Pagination : 35-57

ISBN : 979-10-90426-27-6

ISSN : 0765-0752

Référence électronique

Marion Lièvre, «Roms roumanisés, Ciurari, Ursari : ethnicité et appartenances sociales. Ethnographie des migrants roms roumains à Montpellier », Revue européenne des migrations internationales [En ligne], vol. $32-n^{\circ} 1$ | 2016, mis en ligne le 01 mars 2018, consulté le 14 avril 2022. URL : http:// journals.openedition.org/remi/7625; DOI : https://doi.org/10.4000/remi.7625 


\title{
Roms roumanisés, Ciurari, Ursari : ethnicité et appartenances sociales. Ethnographie des migrants roms roumains à Montpellier
}

\author{
..: Marion Lièvre ${ }^{1}$
}

Ceux que le sens commun désigne largement par le terme de "Roms " sont avant tout des citoyens d'un État-nation, soit des Roms roumains, Roms français, etc. Et dans chacun de ces pays, le terme "rom " est souvent décliné par les intéressés en une multitude d'endo-ethnonymes et d'hétéro-ethnonymes. En référence aux Roms de Roumanie, s'ouvre en général un large panel d'ethnonymes et d'hétéronymes : Cortorar, Lăieş, Ursar, Lăutar ${ }^{2}$, Roms roumanisés, etc. Cette contribution propose d'interroger l'usage que les Roms ${ }^{3}$ font de ces catégories d'identification ${ }^{4}$ à partir des observations effectuées auprès de trois groupes de migrants roms roumains présents à Montpellier : les Popescu ${ }^{5}$ ou Roms roumanisés, les Rocşa dits Ciurari ${ }^{6}$ et les Ursari dits Bogdăneşteni. Que signifie se déclarer "Rom roumanisé " ou encore que traduit la désignation d'un autre groupe de Roms comme Ciurari ? Que révèle la mobilisation de frontières par ces groupes pour se définir et identifier les autres groupes, quant aux logiques de l'appartenance (Barth, 1995) ? Quel rapport à la culture émerge de ces processus?

\footnotetext{
1 Docteure en ethnologie, post-doctorat projet ANR " Marg-In ", CITERES, Université François Rabelais de Tours ; marionsli@hotmail.com

2 Cortorar: litt. qui vit en tente (cort = tente). Cortorari [pl.] ; Lăieş: litt. Tsigane nomade. Lăieşi [pl.] ; Ursar: litt. montreurs d'ours. Ursari [pl.] ; Lăutar: litt. ménétriers, violoneux. Lăutari [pl.]. désigne un groupe de Roms musiciens. Ces quatre ethnonymes/hétéronymes ne renseignent en rien aujourd'hui la pratique professionnelle exercée ou le type d'habitat des groupes tsiganes usant ou étant désignés comme tel.

3 J'utiliserai pour cet article le terme "Rom ", reconnu officiellement en Roumanie, lorsque j'évoque les "Roms " d'un point de vue institutionnel ou lorsque je reprends les catégories de la pratique des représentants roms roumains. A l'inverse, je reprendrai les diverses catégories utilisées par les différents groupes étudiés lorsque je me situerai dans l'analyse de leurs propos. Ces catégories peuvent être "Tsigane ", "Ciurari ", "Bogdăneşteni ", "Roms roumanisés ", etc.

4 Sur la question des processus d'identification, voir Avanza et Laferté (2005).

5 Tous les noms de famille, de village et les hétéronymes ont été modifiés.

6 Litt. "Ciurar " [pl. ciurari] signifie littéralement " criblier ", et ciur: " tamis ", " crible ".
} 
La question des ethnonymes et hétéronymes des groupes roms peut paraître largement rebattue. Elle est presque toujours de vigueur dans les écrits sur les Roms, car elle traduit un positionnement théorique : essentialisme versus constructivisme ${ }^{7}$. L'idée de l'existence d'une diversité de groupes roms est communément admise. La valorisation de cette diversité en singularité - la nation rom - divise ${ }^{8}$. Étudier les usages et les frontières des catégorisations ethniques participe d'une approche constructiviste déjà initiée par les chercheurs étudiant les "diversités roms " (Hasdeu, 2007 ; Olivera, 2012 ; Cossée, 2010). Pour autant, ces processus n'ont pas vraiment fait l'objet d'une lecture centrée sur les enjeux et rapports de force que ces catégories traduisent entre les groupes roms du point de vue des logiques de l'appartenance ethnique et sociale. Or l'analyse des catégories ethniques apparaît être un bon point de départ pour comprendre le fonctionnement de l'ethnicité et les logiques d'appartenance en jeu, comme le soumettait Barth (1969). Fruit d'une construction (Brubaker, 2001 ; Amselle et $\mathrm{M}^{\prime}$ Bokolo, 1985) et d'une négociation constante (Poutignat et Streiff-Fénart, 1995), elles traduisent une forme d'organisation sociale (ibid.) autant qu'elles désignent " la manière dont les acteurs sociaux pensent les divisions et les inégalités sociales en terme d'appartenance et de différenciation ethniques" (Géraud et al., 2002 : 66). Centrale dans la sociologie de la migration (Rea et Tripier, 2008 ; Streiff-Fénart et Poutignat, 2008 ; etc.), l'étude de l'entremêlement des logiques d'appartenance sociale et ethnique se fait plus discrète dans les travaux sur les migrants roms roumains dans le champ de la recherche française. Ils traitent davantage de l'analyse des interactions entre politiques publiques et ses bénéficiaires (Legros et Vitale, 2011 ; BenarroshOrsoni, 2015 ; Clavé-Mercier, 2015 ; Cousin, 2009 ; Lièvre, 2014), appréhendent la migration comme phénomène migratoire dans sa globalité (Benarrosh-Orsoni, 2015), ou encore abordent le processus d'ethnicisation de la " question rom " en France (Olivera, 2011 ; Fassin et al., 2014).

Si les observations de terrain auprès des migrants roms roumains $\mathrm{m}^{\prime}$ ont amenée à privilégier l'étude de la migration en termes de frontières ethniques, de relations inter-groupes et non pas en tant que phénomène migratoire ou d'analyses des politiques publiques c'est parce qu'elles sont venues nourrir ma réflexion sur la déconstruction du rapport à la culture et à l'ethnicité des Roms en Roumanie post-communiste et multiculturaliste engagée à partir d'un groupe "lăutari » de Bucarest (Lievre, 2013). Complémentaire, la situation montpelliéraine initiait en perspectives. Ma présence depuis 2009 en tant que bénévole/ traductrice auprès de différentes familles - au gré d'accompagnements dans des démarches de tout ordre ${ }^{9}-m^{\prime}$ a permis de constater de forts processus de différenciation entre des groupes roms roumains, aux origines géographiques, sociales et ethniques différentes. Les processus de catégorisation prenaient sens

\section{Voir chapitre 1, Olivera (2012).}

8 Voir les débats dans les années 1980 opposant des chercheurs engagés dans la valorisation des singularités culturelles et de leur étude (Williams, 1993; Reyniers, 1992 : Piasere, 1985 ; etc.) et d'autres privilégiant l'existence du peuple rom et d'une unité intrinsèque aux différents groupes (Liégeois, 1976 ; Auzias, 1995), etc.

9 Démarches judiciaires, scolaires, sanitaires, administratives, etc. L'observation participante caractérise principalement ma démarche d'enquête, largement alimentée de discussions informelles lors des accompagnements et d'entretiens plus formels. 
au sein d'interrelations quotidiennes, d'un microcosme rendant probant l'impact de la position sociale sur l'ethnicité.

En effet, ces données ont été récoltées auprès de trois groupes originaires de deux régions et trois lieux différents, mais qui par la situation migratoire se trouvaient placés dans une proximité géographique - habitent dans le même quartier montpelliérain - et sociale : ils ont tous trois fait l'objet de politiques d'évacuation, et deux d'entre eux de relogement toléré. La présente contribution porte sur l'analyse de plusieurs catégorisations "Roms roumanisés ", "Ciurari ", "Cortorari ", "Bogdăneşteni " ou encore "Roms traditionnels " mobilisées par trois $^{10}$ des quatre groupes fréquentés : les Rocşa dits Ciurari ou Roms traditionnels, les Popescu dits Roms roumanisés et les Ursari dits Bogdăneşteni.

Tableau 1 : Ethnonymes et hétéronymes par appartenance familiale et régionale

\begin{tabular}{|l|l|l|l|}
\hline \multicolumn{1}{|c|}{ Noms de famille } & \multicolumn{1}{|c|}{ Ethnonymes } & \multicolumn{1}{c|}{ Hétéronymes } & \multicolumn{1}{c|}{ Région } \\
\hline Popescu & Roms roumanisés & Kastalé* & Transylvanie \\
\hline Rocşa & Cortorari & $\begin{array}{l}\text { Ciurari, Roms } \\
\text { traditionnels }\end{array}$ & $\begin{array}{l}\text { Bogdăneşteni, } \\
\text { Bucureşteni, Roms } \\
\text { traditionnels }\end{array}$ \\
\hline $\begin{array}{l}\text { Plusieurs noms de } \\
\text { famille }\end{array}$ & Ursari & Valachie \\
\hline
\end{tabular}

* Le terme Kastali (Kastaliu au singulier) désigne les " Roms assimilés linguistiquement et en termes de mode de vie [...] issu du mot Kast, bois en langue romani " (Gheorghe, 2012 : 306). Il revêt différentes orthographes. Orthographié ainsi, il est celui utilisé par les représentants roms roumains ; on rencontre aussi Kasthali, Casthali.

Cet article se propose d'interroger l'usage des catégories mobilisées par les intéressés. II ne s'agit donc pas de proposer une ethnographie de l'un de ces groupes. J'interrogerai dans un premier temps, I'usage des catégories "Rom roumanisé " et "Ciurari " (cas des Popescu et Rocşa). Qui sont les Roms qui mobilisent ces catégories et que traduisent les marqueurs posés comme frontières entre ces deux groupes? Dans un second temps, je focaliserai davantage sur les marqueurs de distinction comme révélateurs d'une certaine forme d'organisation sociale (cas des Rocşa et Bogdăneşteni). Enfin, j'analyserai I'enjeu des logiques de distinction entre les différents groupes sur la cristallisation d'un certain rapport à la culture. De manière transversale, I'article interroge l'impact du contexte migratoire sur ces processus de catégorisations en comparant avec les observations effectuées en Roumanie.

10 La classification des migrants roms roumains en trois groupes repose sur le critère de I'origine géographique et non de l'installation par terrain : en effet, Montpellier comptait alors six platz (terme utilisé par les migrants roms roumains pour désigner leurs lieux de vie) et un squat. Le nombre de terrains est mouvant au gré des expulsions, relogement, etc., mais les familles présentes sont toujours les mêmes à quelques variations près. Les changements dans I’habitat n'ont pas influé sur les divisions entre eux. 


\section{Des " Roms roumanisés ": éloignés de valeurs culturelles?}

\section{Présentation des familles : \\ Popescu/u Roms roumanisés " versus "Ciurari "/Rocşa}

Dès les premières rencontres avec les Popescu, ils me précisent de manière récurrente qu'ils sont " différents des autres Tsiganes, car ils sont plus roumanisés ". Ils se disent " Roms roumanisés".

Par "Popescu ", je me réfère à la famille Popescu. Elle rassemble autour des parents Popescu, cinq enfants aujourd'hui adultes et mariés, dont trois fils et deux filles. Le corpus de données analysées a été récolté auprès de Vlad, la quarantaine - enfant aîné Popescu -, sa femme Dorina, Lena et Violeta, filles Popescu (cf. Schéma 1). Ces trois enfants et leurs familles vivent avec leurs parents sur un terrain que la mairie leur a octroyé en octobre $2010^{11}$. Chacun d'eux s'est aménagé son chez soi, alliant mobile home à une extension en bois, s'ouvrant sur une terrasse sans vis-à-vis. Les deux autres fils sont installés en appartement et les visitent régulièrement.

\section{Schéma 1 : Famille Popescu}

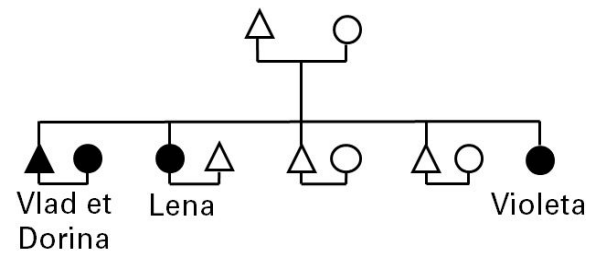

Les Popescu utilisent la catégorie "roumanisé " surtout lorsqu'ils parlent des Rocşa. Les Rocşa ont également été relogés ${ }^{12}$ à deux mois d'intervalle des Popescu, sur un terrain situé en vis-à-vis dans un quartier du nord-ouest de la ville, suite aux négociations du collectif avec la mairie de Montpellier. Les propos des Popescu vis-à-vis des Rocşa sont à recontextualiser dans le cadre de cette politique de relogement, car ce sont les deux seuls groupes parmi les trois évacués en août 2010 à Montpellier à être relogés alors que pour les Popescu, les

11 Les Popescu ont été expulsés du squat où ils s'étaient installés en août 2010. Suite à cette expulsion, le collectif de défense des Roms créé à Montpellier cette même année, a sollicité la mairie pour un relogement. Cette sollicitation a abouti à la mise à disposition d'un terrain pour ces familles en octobre 2010.

12 Fin 2010, les Popescu investissent le premier terrain et début 2011, les Rocşa s'installent sur un second. Ces deux relogements ont consisté en la mise à disposition d'un terrain vierge, l'installation de sanitaires, d'un panneau électrique et d'un point eau. La mairie a imposé I'habitat en caravanes et a accepté de payer une partie du prix des caravanes. 
Rocşa ne devaient pas bénéficier d'une attention similaire de la part des pouvoirs publics au vu de leur "volonté d'intégration " en France. C'est ce climat de luttes symboliques pour l'accès aux droits qui va en partie favoriser ou du moins alimenter un processus de distinction chez les Popescu (Bourdieu, 1980 et 1998).

Les familles Rocşa, de leur nom de famill,e sont désignées par les Popescu par l'hétéronyme "Ciurari " ou "Roms traditionnels ", j'y reviendrai. Rocşa est le nom de famille porté par plusieurs groupes familiaux élargis ayant des liens de parenté entre eux, présents à Montpellier et vivant tous sur un même terrain. Par Rocşa, je me réfère ici à l'un de ces groupes élargis, le plus conséquent au moment de l'enquête, qui comporte le couple parental, M. et Mme Rocşa et leurs six enfants, tous mariés et parents à ce jour. Les enquêtes ont plus particulièrement été menées avec trois de ces enfants: Sorina, aînée de la famille, Ilie, fils aîné et sa femme, Viorela, Doru et sa femme Stefania (cf. Schéma 2). Chaque noyau familial de cette famille élargie est installé avec sa famille dans une colibă [litt. cabane ${ }^{13}$ ]. Toutes adossées les unes aux autres, elles forment un rectangle avec au centre, un grand terre-plein. Chaque colibă possède une ouverture extérieure vers l'intérieur de l'espace habité. Cet espace forme un lieu public et commun partagé par tous et au regard de tous. Cette famille élargie compte environ vingt-cinq personnes.

\section{Schéma 2 : Famille Rocşa 1}

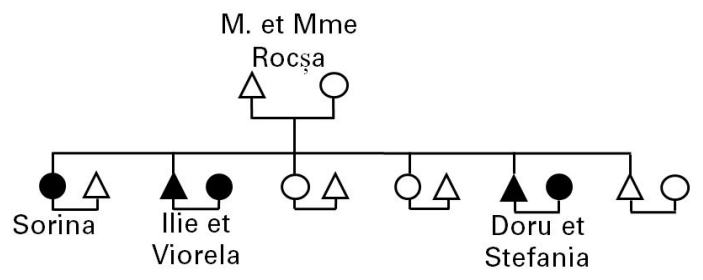

\section{Roumanisés versus Ciurari : des différences posées comme culturelles}

L'identification des Popescu comme "Rom roumanisé " par distinction d'avec les Rocşa dits "Ciurari ", se traduit par l'évocation de différences de " coutumes" - pour reprendre leurs termes - et occupe une part importante de leurs conversations quotidiennes. Les Popescu évoquent ne plus parler le romanès, ou encore ne pas partager les codes "culturels " des " autres Tsiganes ", comme le mariage des enfants en bas âge, la tenue vestimentaire, mais aussi, les codes culinaires. Par exemple, lors des jours de fêtes, ils mettent en avant la spécificité de leurs mets, qu'ils notent être de meilleur goût. Aussi,

13 Terme que les migrants roms roumains utilisent pour désigner leurs habitats en France lorsqu'il s'agit d'une construction ou d'une extension bricolée avec des matériaux de récupération. 
chaque fois que nous parlons cuisine, les femmes Popescu aiment insister sur le fait que " chez eux [entendu leur groupe familial], le batik ne se porte pas", et encore moins "le tablier ", comme le font les autres Tsiganes d'ici. Elles sont notamment fières de mentionner que leurs maris ne les obligent pas à porter des jupes, et qu'elles sont libres de s'habiller comme elles le souhaitent. À écouter les Popescu, les Rocşa apparaissent d'emblée comme les Tsiganes qui ont " conservé la culture " ${ }^{14}$, ceux que le sens commun apparente en général à la catégorie des "Tsiganes traditionnels ". Les Popescu, à l'inverse se définissent comme éloignés des valeurs culturelles qu'ils identifient chez d'autres Tsiganes et recourent dans ce sens au qualificatif " roumanisé " pour s'identifier. "Roumanisé " renvoie ici à la question de l'ethnicité ou à la question des critères ethniques d'appartenance entendus dans un sens essentialiste : je suis Rom, car je possède des caractéristiques culturelles (langue, tenue vestimentaire, coutumes, danses, etc.).

Le recours à des caractéristiques culturelles dans la définition de soi est quasi-omniprésent chez les Roms de Roumanie. Les Lăutari se désignent avant tout comme "Roms roumanisés " en prenant soin de préciser ne pas parler le romanès, ou encore se distinguer dans la tenue vestimentaire ${ }^{15}$. On retrouve également cet usage chez les activistes roms auprès desquels j'ai mené des enquêtes de terrain à Bucarest. Persida, en deuxième année d'études de romani à I'université de Bucarest au moment de l'enquête, m'explique en guise de présentation, I'ambiguïté de son appartenance ethnique. Elle est issue d'" une communauté " qu'elle ne peut qualifier de "traditionnelle ", car précise-t-elle " nous n'avons pas le costume chez nous. Bon, notre neam ${ }^{16}$... nous sommes Ursari [litt. "montreurs d'ours"] et nous avons seulement la langue, seulement ça, nous parlons la langue mais le costume, je me réfère au vestimentaire, il ne s'est pas conservé. Bon, beaucoup de coutumes se sont conservées mais beaucoup d'autres non, seulement la langue ".

Ainsi les catégories "roumanisé " et " traditionnel " sont mobilisées pour traduire une appartenance culturelle. Les Popescu n'évoquent pas seulement des critères identifiés comme "culturels " lorsqu'ils évoquent être " roumanisés ". Comme le souligne Cuche (2004 : 95) à propos de Barth (1995), " pour expliquer l'ethnicité, ce qui importe, ce n'est donc pas d'étudier le contenu culturel de l'identité, mais plutôt les mécanismes d'interaction ". C'est dans ce sens que les critères mobilisés par les Popescu m'ont interpellée.

14 À propos de la déconstruction de la notion de culture, comme héritage ancestral, Olivera (2012) montre, que ce qui est désigné comme "tenues traditionnelles " chez les Gabori de Transylvanie, n'est rien d'autre que la reprise des tenues paysannes roumaines des années 1970, 1980.

15 Pour plus de précisions, se reporter au chapitre 8 de ma thèse de doctorat (2013) et aux travaux de Rădulescu (2004) et Beissinger (2001).

16 Neam est ici utilisé dans le sens de "groupe de parenté " et de manière plus large, ce groupe peut être entendu dans un sens ethnique, les Ursari. 


\section{Roumanisé : traduction d'un processus de distinction sociale}

Ce qui transparait à travers ces jeux de " distinction culturelle ", c'est l'utilisation de jugements moraux, de jugements de valeurs à propos de ce que les Popescu désignent comme les " coutumes " (obiceiuri) des Ciurari. Notamment, lorsque Lena me raconte la singularité de leurs coutumes culinaires, ce qu'elle met en avant, c'est une différence dans la manière de recevoir. M'évoquant le récent baptême célébré chez les Popescu, auquel les Ciurari étaient invités, elle me raconte amusée et touchée que ces derniers se sont plaints de la faible quantité de nourriture, ne comprenant pas I'usage du hors-d'œuvre. II apparaît clairement que Lena exprime ici une distinction de classe. Distinction d'autant plus illustrée lorsqu'au lendemain d'un mariage chez les Ciurari, les Popescu se moquent avec dédain de leur manière de cuisiner la nourriture et de leur service " à même le sol ". Déclarant ces prestations conformes aux "coutumes " des Ciurari, un regard porté sur le capital culturel sous sa forme institutionnalisée soit les titres scolaires - en donne une tout autre lecture. Ces processus de distinction traduisent avant tout ce que Bourdieu (1979) a conceptualisé comme une différence d'habitus. En effet, sur les six enfants Rocşa, seul Ilie a fréquenté l'école et peu de temps, à cause de son côté " bagarreur " aime-t-il raconter. Tous les enfants Rocşa sont analphabètes. En France, seul un enfant des six enfants Rocşa était scolarisé au moment de l'enquête. À l'inverse, chez les Popescu, les femmes ont terminé le cycle secondaire et les hommes le premier cycle du lycée professionnel (I'équivalent de la classe de première en France). Tous les enfants Popescu ont donc été scolarisés et scolarisent aujourd'hui leurs enfants, à partir de la maternelle.

Aussi, la référence au port du batik et du tablier chez les Ciurari, illustre dans un sens l'urbanisation des Popescu et l'émancipation sociale qui s'en est suivie. La différence marquée ici est empreinte de l'opposition ruralité/urbanité où le batik et le tablier renvoient à des pratiques rurales liées notamment à la condition de la femme. Issus d'une petite ville, les Popescu se sont vite urbanisés en s'installant dans la préfecture du département, Brad. Le père de famille, Mihai, décide d'y acheter une maison pour échapper aux conflits avec les autres Tsiganes d'Aiud dont certaines familles Rocşa, trop nombreuses, précise-t-il. La famille s'installe à Brad au milieu des années 1980, dans un quartier hongrois résidentiel, aime-t-elle spécifier. À l'inverse, les Rocşa sont pour la majorité installés dans un petit village de moins de 3000 habitants, à côté d'Aiud, traversé par une route principale et quelques rues adjacentes. Tous les membres de la famille élargie "Rocşa " habitent ensemble sur une parcelle dans une même rue, perpendiculaire à la rue principale qu'ils nomment ţiganie.

Les logiques de distinction posées par les Popescu mettent en lumière deux trajectoires sociales opposées. Les uns, d'un milieu rural, sans scolarité, les autres, d'un milieu urbain et scolarisés. Le capital culturel n'est pas le seul élément de distinction mobilisé par les Popescu. C'est notamment ce que traduit l'usage du terme "Ciurari " pour désigner les Rocşa. Souvent employé avec malice et amusement, parfois mis en scène avec dédain pour imiter l'activité de criblier que ce terme signifie, il connote pour les Popescu une manière de hiérarchiser les statuts professionnels entre activités artisanales et salariées et fait donc écho à la possession d'un capital économique (Bourdieu, 1980). En effet, l'évocation du terme Ciurari s'accompagne d'une comparaison avec d'autres métiers 
artisanaux souvent attribués à des groupes tsiganes "căldărari ", " spoitori " [étameurs] et d'une précision sur " ce que signifie pour les Popescu travailler". Ils posent l'artisanat comme une activité différente du travail et l'excluent de ce qu'ils considèrent travailler : "Ils ne savent pas ce que c'est de travailler, car ils n'ont jamais travaillé ". L'usage de cet ethnonyme traduit davantage la volonté de se démarquer professionnellement qu'il n'indique l'activité professionnelle des Rocşa. Et c'est notamment ce que confirme la comparaison du statut professionnel de ces deux groupes familiaux. Selon la classification roumaine des professions et catégories socioprofessionnelles (PCS $)^{17}$, sous le régime communiste $^{18}, M$. Rocşa dont l'activité relève du domaine agricole, est ouvrier agricole non qualifié dans les entreprises ou coopératives agricoles de production (IAS/ $\mathrm{CAP}^{19}$ ) et $\mathrm{M}$. Popescu dont l'activité relève du milieu industriel, est ouvrier qualifié (chauffeur et ses fils, mécaniciens) ${ }^{20}$. Je me réfère ici aux activités des parents de la famille Popescu et Rocşa, soit la première génération sur les trois présentes en France, car la deuxième génération n'était pas en âge de travailler jusqu'en 1990. L'aîné de la famille Popescu avait seize ans en 1989, celui de la famille Rocşa, douze. Cette différenciation de statuts professionnels se vérifie aussi en France, eu égard aux activités pratiquées par chacun des membres de ces deux familles. Les Rocşa - hommes et femmes confondus - pratiquent pour la majorité la mendicité. Les fils Popescu, mécaniciens de formation, ont mis à profit leur savoir-faire en proposant leurs services aux autres Tsiganes présents à Montpellier mais aussi aux populations locales. L'achat, la revente, en passant par la réparation, caractérisent le quotidien de Vlad qui continue à récupérer de la ferraille, après être passé quelques années, lui aussi, ainsi que sa famille, par la mendicité.

Les critères mobilisés par les Popescu lorsqu'ils évoquent les Rocşa sont des marqueurs de capitaux culturel et économique, et apparaissent comme des facteurs déterminants de la position dans l'espace social et la hiérarchie sociale (Bourdieu, 1998), plutôt qu'ils ne renseignent leur rapport à la culture. Le qualificatif " roumanisé " ne renvoie donc pas à une " perte ou un éloignement des caractéristiques culturelles " mais bien plutôt à un processus de distinction sociale. En cela le qualificatif "roumanisé " ou le terme Ciurari ne se réfère pas ici à des "catégories ethniques " mais à des " catégories sociales ". C'est notamment ce que traduit la précision faite par Vlad: " nous sommes "Tsiganes roumanisés" ou encoreTsiganes "de mătase" [de soie] ou "domneşti " [princier], car "la soie est de meilleure qualité que le coton" ". Le choix de ces qualificatifs renvoie dans ces deux cas à une qualité noble, marque d'une volonté de se

17 Pour la classification des professions en Roumanie, se référer au site Internet du recensement : http://www.recensamantromania.ro/wp-content/uploads/2011/03/PROIECT_ NOMOC_listare.pdf

18 M. Rocşa gardait des animaux - moutons, vaches - pour un Roumain et en avait également chez lui. Ilie l'aidait, " mes parents m'ont élevé avec les vaches ", s'amuse-t-il à dire lorsque je lui demande s'il est allé à l'école. Cependant, les enfants Rocşa n'ont jamais travaillé de manière "salariée " ou "formellement " depuis la fin du communisme.

19 IAS et CAP sont les sigles utilisés sous le communisme pour désigner les entreprises et coopératives agricoles d'État.

20 Le père Popescu, Mihai était chauffeur pour I'entreprise de ménage de la ville IGOD [Întreprinderea de Gospodărie Orăşenească Deva] et sa femme, employée de ménage. Les fils Popescu ont ensuite été embauchés dans I'usine où travaillait leur père, notamment en tant que mécaniciens. 
distinguer socialement. C'est également selon ce même rapport social (capital culturel), mais inversé, que les Rocşa se représentent les familles Popescu. Ilie, aîné de la famille Rocşa considère qu'en plus de sa connaissance de la langue romanès, s'il savait lire et écrire, il serait un " Rom noble " [domnesc].

Les Roms qui se désignent "Roms roumanisés " ne sont donc pas plus " acculturés " que ceux qui se désignent ou sont désignés comme " traditionnels ". Il ne s'agit pas de postuler que seule la position sociale les distingue, mais de mettre en avant que les processus de catégorisations ethniques ne renseignent pas l'ethnicité, qui repose davantage sur une forme d'organisation sociale, que sur le partage de caractéristiques identitaires (Barth, 1969). La manière dont ces groupes vivent leur " tsiganité " diffère sans pour autant s'exclure et les Popescu ont notamment leur manière de vivre leur "tsiganité " tout comme les Rocşa.

Bien que ces logiques de distinction traduisent des appartenances sociales, les marqueurs posés sont eux des références à des caractéristiques identitaires (tenue vestimentaire, danse, etc.) et traduisent d'une certaine manière la présence à la référence culturelle chez les Popescu. Ce constat nous amène à interroger l'impact du contexte migratoire : renforce-t-il ces logiques ou au contraire, lui pré-existent-elles sous cette même forme ? Les travaux effectués pendant la thèse auprès de Roms Lăutari en Roumanie permettent d'esquisser quelques réponses. Les observations ont montré que les Lăutari avaient le même usage de la catégorie "roumanisé " que les Popescu, à l'exception de quelques critères posés comme marqueurs de distinction propres au contexte de socialisation. Pour s'ériger au rang de "Roms roumanisés ", les Popescu se distinguent des migrants roms montpelliérains au capital culturel et économique moindre que le leur, alors que les Lăutari se réfèrent à d'autres Roms roumains fréquentés au quotidien désignés comme "proşti crescuţi " [mal élevés] (Lievre, 2013). Ces logiques sont donc alimentées dans chaque cas par le contexte de socialisation et s'observent hors contexte migratoire. On peut maintenant se demander s'il les favorise et si oui, de quelle manière ? La présence de ces processus de distinction chez les Popescu permet d'affirmer que la migration participe de leur constance. Le contexte politique d'observation est également une clé de lecture de la manière dont ces processus émergent. C'est en effet dans un contexte quotidien de luttes pour l'accès aux droits qu'ils ont été observés. Sous-tendues par des considérations homogénéisantes "les Roms ne veulent pas s'intégrer ", les politiques publiques visant les Popescu au moment de l'enquête, oscillent entre évacuation et relogement avec sélection des personnes ${ }^{21}$ (Legros et Vitale, 2011). Dans ce sens, elles alimentent un processus de distinction et de positionnement social.

\footnotetext{
21 Les Popescu et les Rocşa ont fait l'objet d'une politique d'évacuation ordonnée par la préfecture de Montpellier suite à la déclaration de M. Sarkozy, sur la sécurité, prononcée au conseil des ministres, le 21 juillet 2010 au palais de l'Élysée. Sur la base d'un constat de "problèmes de comportements " attribués aux " Gens du Voyage " et aux "Roms ", deux groupes distincts par leurs origines nationales, M. Sarkozy déclarait la nécessité des "expulsions de tous les campements en situation irrégulière ". C'est dans ce contexte politique d'homogénéisation et de généralisation que les Popescu se sont distingués des Rocşa, mettant en avant notamment ce que j'ai désigné comme " un profil de l'immigré intégré " (Lievre, 2014).
} 
Enfin, les analyses faites auprès des Lăutari en Roumanie confirment la prégnance des logiques de distinctions sociales, qui apparaissent bien plus un enjeu pour les Roms roumains de manière générale que l'identification ethnique $^{22}$. Ceci étant, ces processus de distinctions sociales participent à la réification d'un " nous ethnique " et nous amène à notre deuxième point de la réflexion : I'analyse des critères de différenciation posés par les Rocşa vis-à-vis d'un autre groupe tsigane présent à Montpellier, les Bogdăneşteni.

\section{Se dire "Cortorari ": " nation prestigieuse " versus " nation inférieure "}

\section{Présentation des familles: Rocşa/Cortorari versus Bogdăneşteni/Ursari}

J'ai abordé les Rocşa sous l'angle de leur profil socio-économique ${ }^{23}$, soit leur position sociale, car c'est notamment ce que renseignait l'analyse de l'hétéronyme "Ciurari", mobilisé par les Popescu. Je propose de poursuivre en partant de l'analyse de l'ethnonyme "Cortorari ", mobilisé par les Rocşa notamment dans un processus de distinction vis-à-vis des Bogdăneşteni. Ici, I'observation des critères de distinction éclaire davantage sur une forme d'organisation sociale, sur les codes et valeurs moraux qui régissent leur appartenance à leur neam, liant étude des frontières et regard sur l'organisation sociale. Et c'est dans I'interaction que cette singularité s'apprécie, contrairement aux idées reçues sur I'isolement comme facteur de reproduction culturelle (Barth, 1969).

La famille Rocşa à laquelle je fais référence ici est une deuxième famille élargie Rocşa. Il s'agit de la famille de la sœur de M. Rocşa, Olimpia, installée sur le même terrain que son frère à Montpellier, avec son mari,Teodor Rocşa, et trois de ses cinq enfants (deux d'entre eux vivent à Grenoble). Les propos rapportés ci-dessous sont ceux d'Alba, fille cadette, non mariée au moment de l'enquête (cf. Schéma 3).

Bogdăneşteni est un gentilé. Il désigne le nom des habitants d'un village roumain, Bogdăneşti situé en Valachie, à l'opposé du village des Rocşa, localisé en Transylvanie. Ils se sont connus en France. Tous les habitants de ce village présents en France sont désignés par les autres Roms, par ce nom de ville ou alors comme "Bucarestois ". Eux-mêmes utilisent parfois l'ethnonyme Ursari pour s'identifier. J'utiliserai Bogdăneşteni lorsque je mettrai en scène les propos des Rocşa à leur égard, et Ursari lorsqu'à l'inverse, je me placerai dans leur perspective.

\footnotetext{
22 Les catégories "Roms traditionnels ", " Roms roumanisés " viennent nourrir le rapport social nécessaire à la production de leur ethnicité, comme l'observe également Beissinger (2001 : 49) : " La construction de l'identité romani parmi les musiciens est un processus social élaboré, impliquant différents modes de négociations, pas contraire à ceux que les autres groupes engagent indubitablement, notamment ceux qui se trouvent également à la frontière entre l'ethnicité et la classe ".

23 Le titre de ce deuxième chapitre provient d'une discussion avec les familles Rocşa le 24 mai 2011 : "Naţia buna/bogată " versus " naţia slabă/mai jos ".
} 
Schéma 3 : Famille Rocşa 2

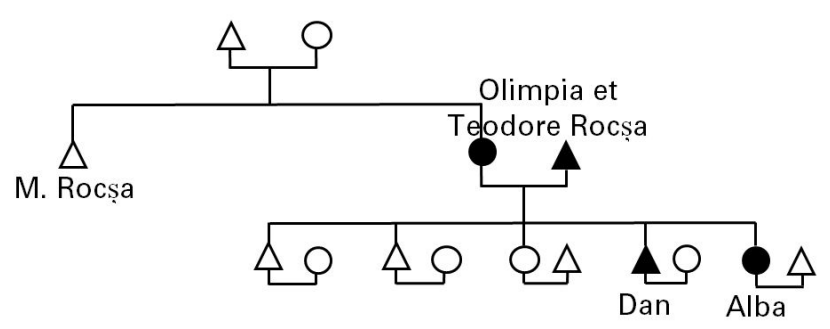

Ce gentilé rassemble différents groupes familiaux, aux liens de parenté comme sans liens de parenté, aux activités diverses et au capital symbolique varié. Ces disparités se retrouvent notamment dans leur répartition spatiale dans le lieu de migration : les Bogdăneşteni occupent plusieurs lieux de vie. Chacun de ces lieux de vie rassemble des familles aux statuts socio-économiques variés. De manière générale, à chaque fois, on peut identifier une famille élargie au capital économique et culturel élevé (cette famille peut-être responsable de terrain ${ }^{24}$ ).

\section{Ce que la relation des Rocşa aux Bogdăneş teni traduit}

À plusieurs reprises lors de discussions avec les membres de la famille Rocşa, le terme Cortorari comme auto-identification est revenu, parfois en précisant que " c'est comme cela qu'on nous désigne en Roumanie ", parfois, présenté comme ethnonyme. Cet ethnonyme et hétéronyme n'est jamais prononcé seul dans une discussion par les Cortorari. Il est toujours accompagné de discours sur les autres "races" deTsiganes, mais aussi de valeurs morales comme " inférieure ", " supérieure ", " riche ", etc. Sur la base de l'analyse d'une discussion sur le mariage avec Alba, fille d'Olimpia etTeodor, et d'autres conversations avec cette famille, il s'agit ici d'interroger ce que signifie l'utilisation de cette " catégorie d'identification " par les Rocşa.

LorsqueTeodor précise être Cortorari, il s'empresse toujours de spécifier qu'il existe " plusieurs races " de Tsiganes différentes, mais aussi " qu'entre eux, ils ne s'aiment pas beaucoup ", désignant les Tsiganes de Bogdăneşti comme exemple. L'auto-identification repose sur la différenciation d'avec d'autres mais pas seulement. Alba, sa fille n'étant pas mariée à vingt-trois ans - ce qui est rare chez les Rocşa - je lui demande pourquoi ne se marierait-elle pas à un Bogdăneşteni, qu'ils fréquentent quotidiennement même s'ils prétendent " ne

24 Par responsable de terrain, j'entends la personne qui trouve le terrain et qui en général est responsable des jeux d'argent que pratiquent ces Roms pour rembourser leurs dettes, contractées pour la plupart depuis leur départ en Roumanie. Ces dettes, qu'ils doivent à des usuriers - pas toujours présents en France et qui ne sont pas directement les responsables de terrain - sont conséquentes. Le principe de la pratique usurière étant que le taux de remboursement augmente une fois le délai de remboursement dépassé (après un mois, la somme est doublée, etc.) 
pas les aimer " ? Alba me répond très fermement qu'il lui est impossible de se marier à un Bogdăneşteni car précise-t-elle : " c'est la honte, tous nos Tsiganes riront ". Et avec un Popescu, relançais-je ? " Ça ne l'est pas, car c'est entre nous, ils sont une autre race de Tsiganes, mais pas si basse ". Plus tard, lors d'un échange similaire à propos de son précédent mariage avec Radu, issu d'un autre neam que le sien, Alba me confirme pouvoir se marier avec des hommes d'un autre neam, à partir du moment où ils appartiennent à une " nation prestigieuse, pas comme celle des Bogdăneşteni, qui est mauvaise [slabă] ".

J'ai montré que l'auto-identification s'accompagne d'une distinction mais cet échange avec Alba traduit un deuxième point : la différenciation est exprimée en termes de hiérarchie. Alba pose ici le mariage comme étant endogame mais surtout homogame : les conjoints peuvent être d'un autre neam, mais ce neam doit être "bon/prestigieux " et non " pauvre/médiocre ". Cette logique de différenciation s'accompagne de qualificatifs (pauvre/médiocre) eux-mêmes appuyés par des valeurs morales qui sont : la honte, mais aussi la peur. En plus d' " humilier leur nation " et de véhiculer du ridicule au sein de son neam, Alba ajoute qu'elle ne peut fréquenter des Bogdăneşteni car " tout le monde a peur d'eux [...], s'en méfient, car ils n'ont peur de rien, ils sont sans gêne ". En d'autres termes, ce qui détermine l'infériorité d'une nation (naţie slabă) pour les Rocşa, c'est de susciter la peur et de ne pas respecter les règles de prestige et de honte : " être sans gêne " [nu ştie de ruşine] et par conséquent "ridiculiser l'autre " [să mă fac de ruşine]. À l'inverse, lesTsiganes caractérisés comme issus d'une " nation prestigieuse " - qu'ils soient Cortorari ou autres - sont décrits comme " respectueux", et avec qui " on peut se comprendre ".

La hiérarchie posée par les Rocşa fait état de valeurs morales et sociales qualifiées par les adjectifs " pauvre/médiocre ", "prestigieux ", " bon ", " respectueux ". Et ces valeurs morales reposent sur des critères comportementaux décriés par les Rocşa : "être inabordables ", ou encore " moqueurs", " mauvais", " des personnes de scandale ", de " conflits ". D'un premier abord, les éléments de distinction formulés par les Rocşa ne concernent pas ce qui a trait à la "culture " : être d'une bonne nation par la maîtrise de la langue de cette nation, ou par I'héritage de valeurs familiales reconnues historiquement ou encore avoir des "traditions" plus respectables que les autres. Avant tout, les critères de distinction s'appuient sur des qualités désignées comme intrinsèques à ce groupe. En d'autres termes, la valeur d'une autre nation pour les Rocşa repose sur le respect de leurs propres valeurs et principes moraux et renseigne par conséquent leur forme d'organisation sociale. Ces observations posent donc les liens de parenté, les valeurs morales de la honte, du respect et de la peur (Ruşinea si frică $)^{25}$ comme constitutives d'une appartenance ethnique au neam cortorari.

La place centrale accordée aux relations de parenté dans ce groupe s'observe à travers différentes pratiques. Les mariages sont endogames et respectent les règles de parenté en vigueur au sein du groupe : on ne se marie pas entre cousins directs (văr primar), mais on se marie avec quelqu'un du même neam, ou du moins d'un neam d'une même valeur que le sien. Les Cortorari

25 L'action des uns est définie par la honte que cette action apporte au groupe : " que leurTsigane ne se moque pas d'eux " (" să nu radă Ţigani noştri de noi »). 
se définissent et s'évoquent les uns les autres par la relation de parenté qui les unit, les membres vivant sur un même terrain partagent tous des relations de parenté proches (frères/sœurs/grands-parents) ou plus éloignées (cousins/ arrière-cousins). La parenté joue aussi un rôle central dans le contrôle social du groupe. Bien que le rapport à l'argent et l'organisation des tâches quotidiennes révèlent l'individualisation et l'autonomie de chaque famille (les bénéfices de la mendicité sont propres à chaque noyau familial nucléaire), les relations entre chaque noyau familial et le rapport aux maladies entre autres, sont révélateurs d'un contrôle social collectif. Les Rocşa s'apparentent à une communauté dans le sens deTonnies (1944), où les relations sociales ne sont pensées qu'au travers des relations de parenté et obligations familiales. Les individus ne sont pas libres et autonomes vis-à-vis des autres membres du groupe. Alors que tous les acteurs associatifs les appréhendent comme " une communauté "-qu'ils nomment aussi "clan " ou " tribu " - par laquelle est entendue un contrôle social régulé par l'autorité des " anciens ", il s'agit de divers groupes familiaux unis par des liens de parenté, dont l'autorité dépend de la place de ce groupe dans le réseau familial et où l'individu est déterminé par l'appartenance et la position de sa famille dans son groupe.

Les valeurs posées vis-à-vis des Bogdăneşteni - " Être ridiculisé par I'autre " [să mă fac de ruşine] ou " ne pas se soucier de la honte ${ }^{26}$ " [nu ştie de ruşine] ou encore la peur que suscitent ces autres, "a fi oamenii buni, cum se căde " [être des gens respectables] - sont omniprésentes dans les expressions quotidiennes des Cortorari qu'il soit question des règles d'alliance, de représentations de certaines maladies, de relations à autrui basées sur le prestige que procure cette relation. À propos des règles d'alliance, Stefania m'expliquait l'importance de la virginité lors du premier mariage par l'expression "se plateşte ruşinea ". "Certaines maladies ne doivent pas être sues, comme le diabète, car elles font des personnes atteintes, des personnes pas respectables pour leur nation ". Enfin, face à leurs demandes explicites de moderniser mes biens matériels comme ma voiture et mon téléphone - je compris l'importance "du prestige " dans leurs valeurs morales. Le respect d'autrui est fonction du prestige apporté par le nouvel arrivant dans la famille, ou de manière plus large dans le neam, et est totalement dépendant de ses biens matériels et de son appartenance socioculturelle.

Mais ces valeurs identifiées comme propres aux Cortorari d'ici vont bien au-delà d'un ancrage lié au territoire et/ou à la situation de migration. Elles traduisent à l'inverse un ancrage ethnique que la situation de migration vient favoriser. En effet, Tesăr (2011 : 309) observait il y a quatre ans à propos de Cortorari parents proches et issus du même village que les Rocşa : " au quotidien, les Cortorari cherchent à obtenir "I'honneur" et à éviter "la honte" ". Le contexte et la situation migratoire apparaissent donc comme propices à la ré-élaboration du sentiment d'appartenance à la nation Cortorari. De même, leurs représentations autour de la pratique de la mendicité telle décrite et observée par Tesăr se retrouvent chez les Cortorari montpelliérains, qui pratiquent la mendicité comme activité principale. Chaque noyau familial se partage le centre-ville de Montpellier, et chacun d'eux possède " son coin " de la ville, où il mendie à

26 Je tiens à remercier Cătălina Tesăr pour m'avoir proposé plusieurs traductions dont celle-ci. 
heure fixe. La mendicité ne rapporte pas beaucoup mais les Cortorari préfèrent cette activité à d'autres, car il leur importe avant tout de "gagner honnêtement de l'argent ", précisent-ils en se démarquant des pratiques des Bogdăneşteni. Ils ont conscience de l'image " dégradante ", de " misérables " que leur confère la mendicité aux yeux des autres groupes tsiganes présents à Montpellier, qui ne manquent pas de le leur signifier. Cependant, pour les Rocşa, la mendicité n'est pas une activité dévalorisante, ou " humiliante " au sein de leur groupe, car elle se conjugue pleinement avec leurs principes d'organisation sociale (prestige et honte) et participe à I'entretien des relations de parenté, au prestige et aux valeurs d'honnêteté de leur groupe. La morale qui accompagne la manière de gagner l'argent a plus de valeur que l'activité en elle-même. Et c'est notamment ce qu'observait Tesăr chez les Cortorari immigrés en Italie : " une activité économique, telle que la mendicité, qui est en général considérée comme une activité dégradante, est pratiquée par les Cortorari sans porter atteinte à leur respect social. Cela est possible dans la mesure où la catégorie de personne chez les Cortorari est basée sur l'évaluation morale du comportement relationnel de l'individu en lien avec les catégories négociables et re-sémantisées de l'âge, du genre et de parenté, dans ce contexte culturel propre " (Tesăr, 2011 : 308-309). La pratique de la mendicité est toujours centrale aux Cortorari de Montpellier, tout comme les valeurs qu'ils lui associent, loin d'une domination symbolique les amenant à s'en distancier. Cette pratique en situation de migration participe ainsi à alimenter les valeurs et normes propres à ce groupe, garantes de l'appartenance au neam, tout comme l'analysait Tesăr (ibid. : 309) : " comme activité qui requiert des connaissances et compétences spécifiques, [elle] occupe une position centrale dans leur économie, et les résultats, loin d'être considérés comme amoraux, assurent de fait la reproduction familiale ".

La situation migratoire vient donc conforter et ré-actualiser la manière dont les Cortorari organisent leur vision du monde social : tout apparaît comme si les autres groupes roms présents à Montpellier participaient pleinement de cette réification de la définition de soi. En effet, les valeurs morales reconnues par les Cortorari à Montpellier fonctionnent au sein même du groupe, et les frontières posées vis-à-vis des Bogdăneşteni viennent conforter et renforcer cet entre-soi, tout comme l'observait Tesăr (ibid.) : " "la honte" est un concept relationnel qui fonctionne seulement à l'intérieur des frontières du groupe et selon les catégories de parenté, d'âge et de genre ". La relation aux Bogdăneşteni et aux autres neam participe ainsi au " maintien " de leur forme d'organisation sociale, de l'autorité sociale de leur neam, de la dignité de leur nation, " d'être des gens respectables ". En d'autres termes, c'est en posant des frontières et en alimentant des processus de distinctions sociales et morales d'avec les autres groupes roms, que les Rocşa maintiennent une appartenance à un « nous ethnique ": " pour que la notion de groupe ethnique ait un sens, il faut que les acteurs puissent rendre compte des frontières qui marquent le système social auquel ils estiment appartenir et au-delà desquelles ils identifient d'autres acteurs impliqués dans un autre système social " (Poutignat et Streiff-Fenart, 1995 : 166). Je l'ai montré avec les Rocşa, mais aussi avec les Popescu, chacune des " nations roms " présentes à Montpellier est intégrée dans des processus de distinction sociale et donc d'auto-identification et en cela, participe à la délimitation des contours d'un espace social ; les lois de cet espace social étant les normes et valeurs propres à chacun des groupes roms présents à Montpellier, comme en témoigne le cas des Rocşa. 
Pour revenir à mon point de départ, la catégorie " cortorari ", j'ai souhaité montrer comment ce terme vient ici préciser une position sociale supérieure mêlée à des principes et à des valeurs moraux propres à une appartenance ethnique, au neam, que la situation de migration vient conforter. Le terme " cortorari " conserve en France une signification heureuse et digne, si on compare avec les observations de Tesăr. Donc, loin de renseigner une appartenance " traditionnelle " figée dans l'espace-temps, comme le renvoie son usage en général, cette catégorie vient avant tout signifier un processus de distinction sociale qui éclaire lui-même un système de valeurs et de principes moraux. Appartenance sociale et ethnique sont indissociables pour comprendre l'usage de ce qui est communément désigné comme " catégorie ethnique ".

\section{Cristallisation autour de caractéristiques désignées comme culturelles et authentiques}

\section{Saillance de caractéristiques culturelles}

J'ai montré que la catégorie "roumanisé " traduit davantage un rapport social qu'une appartenance ethnique, entre les Popescu et les Rocşa. Les termes "roumanisé " et "ciurari " sont respectivement des ethnonymes et hétéronymes qui renvoient à un rapport social ethnicisé ; c'est dans ce rapport social que le rapport à l'ethnicité est défini. Les processus de distinction sociale opèrent on l'a vu par la mise en avant de caractéristiques culturelles moquées mais aussi dépréciées pour se valoriser soi-même. Les logiques de différenciation entre ces deux groupes viennent poser des caractéristiques culturelles comme saillantes dans la définition de soi : la tenue vestimentaire (batik, jupes fleuries), les pratiques culinaires, I'hétéronyme " ciurari ", etc.

Entre les Bogdăneşteni et les Rocşa, on assiste à un processus similaire à une nuance près : les Bogdăneşteni et les Rocşa sont les deux seuls groupes à Montpellier à se distinguer des autres par quelques pratiques propres à leur neam qui sont : parler le romanès au quotidien, les mariages précoces, une tenue vestimentaire liée à un système de valeurs, I'usage d'un ethnonyme : ce que je désignerai sous le vocable de " capital identitaire ${ }^{27}$ ". Cette situation de " face à face identitaire " n'est pas sans répercussion sur ce qui est entendu comme " romanes".

Dans ce jeu de distinction basé sur la construction d'une hiérarchie selon des valeurs et principes propres au neam d'appartenance des Rocşa, les Rocşa déprécient les valeurs culturelles des Bogdăneşteni, comme la langue ou les règles du mariage. Dan m'explique : " chez nous, nous n'achetons pas les filles, c'est une honte, si on en vient à parler de nous dans les journaux. Nous ne sommes pas comme les Bogdăneşteni, eux ils vendent leurs enfants, et ils les vendent chers, environ 3000 euros ils donnent. C'est la honte chez nous de faire ça ". Alba déclare ne pas comprendre la langue des Bogdăneşteni car " c'est une autre nation et ils ont un autre accent ". Ces logiques de différenciation s'appuient sur les valeurs morales propres aux Rocşa et prennent corps

27 C'est-à-dire des valeurs culturelles reconnues par eux-mêmes comme « valeurs identitaires " (langue, coutumes du mariage, tenues vestimentaires). 
dans un rapport plus large de différenciation sociale (nation prestigieuse/nation mauvaise). II n'empêche que le cœur de la différenciation repose sur des caractéristiques culturelles "la langue ", les "règles du mariage ", qui dans ce rapport deviennent cristallisées et naturalisées comme définition de soi (entendu au sens du neam).

On retrouve le même processus lorsque les Ursari évoquent les Rocşa bien qu'ils soient moins enclins à des processus de distinction vis-à-vis des autres Tsiganes montpelliérains. Cependant, au hasard d'une discussion sur la situation locale des autres Tsiganes, les Ursari vont se distinguer principalement d'un groupe, celui des Rocşa. Toujours dans un rapport de distinction sociale similaire à celui des Popescu - souvent rendu explicite par les regards de dégoût et/ou les grimaces pincées qui accompagnent leurs propos - les principaux marqueurs de distinction posés concernent la tenue vestimentaire, leur langue, leurs pratiques économiques et l'hétéronyme. Ainsi les expressions "jupes plissées colorées ", "langue laide ou confuse ", Pletoşi ou encore Lăieşii sont souvent de mises pour évoquer les Rocşa. Feuilletant un livre pour enfants en romani standardisé amené par mes soins, Raluca se demande quelle est cette langue. Après lui avoir spécifié qu'il s'agit de la langue romani, elle m'explique - me prenant différents mots pour exemple - que leur langue est beaucoup moins compliquée que celle du livre, car chez eux, de nombreux mots sont dérivés du roumain. Elle ajoute que cette langue est plutôt celle parlée par les Pletoşi, désignant les familles Rocşa. Raluca use aussi de I'hétéronyme Lăieş, auquel elle associe des conditions de vie misérables, sales, précisant que le terrain où ils vivent est rempli de nombreux rats. Raluca n'est pas la seule " ursăriţă " [femmes ursari] à tenir ces propos. Au cours d'une discussion avec M. Eugen - Bogdăneşteni d'une autre famille que Raluca - nous parlons de ses activités économiques. La mendicité vient à être évoquée. M. Eugen s'offusque et me répond qu'il n'est pas comme les Spoitori (en référence aux Rocşa) qui mendient en centre ville. II me précise qu'il n'a rien à voir avec cette nation, car il est intégré et travaille la ferraille.

Comme dans le cas des Popescu, ces processus de distinction principalement centrés sur des caractéristiques culturelles traduisent une distinction de classe. Les propos rapportés ci-dessus sont ceux de Bogdăneşteni au capital culturel et économique supérieur à celui des Rocşa ${ }^{28}$. Et c'est notamment ce que traduit la vision personnelle d'un mendiant exprimée par Relu, autre Bogdăneşteni récemment installé sur le platz des Rocşa ${ }^{29}$. Comparant les Lăieşi aux mendiants, il les décrit comme " une couche sociale des plus basses " [" la pătură de mai jos "], "sur laquelle tout le monde marche, se permet des injures ", précisant leur non-éducation: "ils n'ont pas fait de classes et laissent leurs enfants toute la journée sans éducation ".

\footnotetext{
28 Eugen et Relu, tout comme quelques autres hommes Bogdăneşteni, ont terminé le cycle secondaire. Les femmes n'ont en général jamais été scolarisées ou leur scolarité s'est arrêtée au cycle primaire. En France, leurs enfants sont scolarisés de manière assidue, I'un d'eux vient de terminer un CAP. Du côté économique, ces familles cumulent droits sociaux et activités informelles comme la récupération de ferraille ou pratique de biffins.

29 Dix mois après l'installation des Rocşa sur le terrain accordé par la mairie, le terrain où Relu loge est évacué sans proposition de relogement. Relu et ses frères vont être les seuls à réussir à s'installer avec les Rocşa.
} 
Dans ce rapport social apparaît donc comme valorisée la référence à l'ethnicité ainsi que la hiérarchisation de valeurs ethniques dans ce processus d'identification à un " nous collectif ". L'usage d'hétéronymes rend compte qu'ils sont aujourd'hui des éléments saillants de leur identification collective, tout comme la langue et la tenue vestimentaire. Au-delà, ces logiques de distinctions apparaissent comme une lutte symbolique entre groupes au même capital " identitaire " pour définir les " valeurs ethniques les plus respectables et authentiques ". En témoigne le rapport à la langue et à la tenue vestimentaire qui deviennent des valeurs identitaires valorisées pour les Ursari, lorsqu'elles sont entendues dans une forme d'altérité marquée (être différent) mais atténuée par la même occasion (parler un romani proche du roumain, porter des tenues différentes mais pas extravagantes). En témoigne également, la naturalisation d'une hiérarchie entre les hétéronymes/ethnonymes (Ursari versus Lăieş).

\section{Au-delà de la saillance de caractéristiques culturelles : la désignation de "Tsiganes authentiques "}

La lutte symbolique entre ces différents groupes pose omniprésent un rapport à la culture et érige certaines caractéristiques culturelles (le romanes, les pratiques de mariage, les tenues vestimentaires, les manières de danser) comme gages de prestige et comme centrales dans la définition de " soi " au sein des migrants roms roumains à Montpellier. Au-delà, les considérations des Popescu tout comme des Rocşa témoignent d'un autre processus : celui de réification d'une authenticité tsigane et interroge quant à la prégnance en situation migratoire de ces catégories de pensées.

Les Popescu reconnaissent chez les Ciurari, les attributs identitaires qu'ils déprécient, tout autant qu'ils les valorisent dans un rapport de définition des valeurs identitaires de leur peuple, dont ils se reconnaissent. Dans leurs descriptions, les Ciurari cristallisent tous les attributs nationaux d'édification de la culture nationale et sont considérés comme les " garants de la tradition tsigane ". Ils sont ainsi également représentés et évoqués, comme emblème de l'identité nationale, comme le sont " les paysans et le folklore " pour les régions françaises ${ }^{30}$. En voici quelques exemples. Le jour du baptême d'un enfant Popescu, plusieurs familles Rocşa sont là. Ovidiu Popescu prenant bien soin de me préciser que "les Ciurari sont d'une autre nation, d'Aiud", me présente leurs danses comme " authentiques ": "I/s dansent les danses tsiganes authentiques alors que nous, nous dansons avec davantage de sérieux, plus noblement [mai domnesc] ". Juste avant le baptême, Lena m'avait conseillée de passer chez les Ciurari pour que les femmes me prêtent leurs tenues afin que je sois habillée en "Tsigane authentique ". Les tenues et danses des Ciurari sont ainsi considérées comme "traditionnelles et authentiques ". Le lendemain du baptême, les échanges vont bon train et se focalisent très vite sur la ressemblance dans la tenue vestimentaire entre les Ciurari et un autre groupe de Roms nommé "Gabori ". Cette base de discussion deviendra vite le prétexte pour discuter des différences entre les nations de Tsiganes chères aux Popescu. II y a environ une centaine de groupes tsiganes, parmi lesquels les "Tsiganes authentiques sont les Ciurari, qui vont avec les charrettes " précise Vlad. Ces différences sont grandes sous divers aspects bien qu'ils aient eu une histoire commune, ajoute-

30 À ce sujet, voir les travaux de Thiesse (1999) et Babadzan (2009). 
t-il. En référence aux Rocşa, Vlad évoque " leurs origines ", notant qu' " eux aussi viennent de là, mais qu'ils se sont modernisés, sont allés vers la civilisation ».

Les valeurs d'un groupe ethnique apparaissent comme étant restées intactes, une sorte d'héritage transmis et conservé par refus de "modernisation ", où la croyance en les ancêtres comme " dépositaires de la tradition " devient centrale. La tradition est appréhendée comme un continuum et de manière opposée à la modernité, "la civilisation ". C'est notamment ce que traduit la précision apportée par Vlad sur la relation entre le caractère traditionnel et l'illettrisme ou le fait d'être " arriérés ". Interrogé sur son rapport à cette " authenticité ", il déclare: "bah, t'es fier d'être Tsigane, mais ça ne signifie pas que tu ne dois pas savoir lire, et que tu dois voler ". La tradition apparaît comme indissociable de l'image de la ruralité, des paysans, constitutive des formes de nationalismes ethnoculturels de la fin du XIXe siècle.

Mais ce processus de domination symbolique n'opère pas que chez les Popescu ; d'autant plus dans ce rapport de domination sociale observé entre les Popescu et les Rocşa, notamment confirmé par la réflexion faite par llie et citée plus haut, sur la noblesse que l'instruction lui procurerait en tant que locuteur de romanès. C'est notamment là que réside un des points forts qu'offre ce terrain : la mise en perspective ou non d'un processus d'intériorisation et donc de domination symbolique des catégories de pensées posées par les dominants (les représentants roms et à Montpellier les Popescu). Plusieurs observations faites auprès des Rocşa éclairent sur les mécanismes d'intériorisation de leur neam comme "les dépositaires d'une tradition ". Les baptêmes ou mariages sont en ce sens exemplaires. Participant à ceux organisés par les Popescu, j'y retrouve à chaque fois les Rocşa et Bogdăneşteni. Ils représentent en cela des moments privilégiés pour observer comment les Rocşa participent de ce jeu en occupant le centre de la piste de danse, offrant des performances posant les participants comme public et spectateur se hâtant devant la danse devenue spectacle. Des Ciurariţe [femmes ciurari] sont convoitées pour la danse par les hommes Popescu, fiers et amusés de pouvoir confronter leur talent à celui magnifié comme " héritage ancestral ". Ce n'est pas pour rien que les Ciurari idolâtrent et s'identifient au film Gadjo Dilo ${ }^{31}$, et notamment auxTsiganes qui y sont représentés. Couramment, cette référence est précisée pour se définir face à un Français, ou pour accompagner une soudaine envie de danser sur des airs d'accordéon, de clarinette rappelant les mélodies des films de Kusturica et Tony Gatlif ${ }^{32}$, loin du style de musique tsigane écouté quotidiennement par chacun d'eux. La référence à l'authenticité est aussi présente dans certains de leurs discours. À l'évocation des autres nations de Tsiganes présentes à Montpellier, Teodor précisait être un " Tsigane authentique " et parler le " vrai ţigăneşte " [la " vraie langue tsigane "].

Ces données montrent que certaines caractéristiques de l'ethnicité telle qu'elles sont vécues par les Rocşa ont été intériorisées comme étant "la tradition des Tsiganes " par les différents groupes roms montpelliérains. Les valeurs sur lesquelles se cristallisent cette " tradition " et cette " authenticité "

31 Tony Gatlif, Gadjo Dilo (1997).

32 Tony Gatlif, Gadjo Dilo (1997) ; Latcho Drom (1993) et Emir Kusturica, Chat noir, Chat blanc (1998); Le temps des Gitans (1989). 
sont culturelles, celles du patrimoine immatériel - la langue, les danses - et matériel - le costume. Ainsi dans ce rapport social et moderne à la " culture ", l'authenticité est définie, mais aussi de manière plus large les "valeurs identitaires " partagées et reconnues par les Roms, tous milieux confondus. Le romanès devient rejeté car il est parlé par les Rocşa dont certains souhaitent se différencier socialement mais il est cependant valorisé comme une valeur "rom ", ainsi que le folklore.

Au-delà, ce rapport à la culture n'est pas sans rappeler les schèmes de pensée des militants roms roumains, pour lesquels l'« identité nationale fondée sur la commune possession d'une culture ", entendue comme " nation culturelle ", constitue le ciment fédérateur ${ }^{33}$ pour " accéder au statut de sujets collectifs " (Babadzan, $2009: 11)^{34}$. Retrouver cette conception moderne du rapport à la culture dans un contexte migratoire et auprès de Roms ne participant pas à la mouvance nationaliste permet de conclure à une forme d'intériorisation de ces schèmes et à un processus de domination symbolique (Bourdieu, 1979), où les catégories de pensée des dominants - leaders roms en Roumanie - deviennent I'enjeu de luttes symboliques chez des Roms, non politisés et émigrés en France. Les déclarations des Popescu comme "roumanisés ", "civilisés " en opposition aux Ciurari considérés comme "leurs ancêtres " et " garants de la tradition ", rendent compte d'un mécanisme de domination symbolique où la vision des représentants roms sur I'" identité rom " devient leur. Cette analyse initie une autre manière de sonder l'impact du mouvement des militants roms sur la constitution d'un sentiment d'appartenance à un " nous collectif ${ }^{35}$. Impact qui, jusque-là, a principalement été appréhendé au travers de la reprise par les Roms non politisés de caractéristiques identitaires comme la langue, l'origine indienne, la mémoire collective ou encore le nomadisme (donnant des résultats bien sûr nuls, les Roms en étant très éloignés). Ces observations offrent donc un nouvel angle d'approche pour jauger de l'intériorisation par les dominés des catégories de pensée des dominants.

33 Les représentants roms roumains militent depuis 1989 en Roumanie et dans un cadre "transnational " - auprès des instances européennes (CE, UE) et internationales (ONU, Banque mondiale, etc.) - pour la reconnaissance de la nation rom, en définissant comme dans tous nationalismes ethnoculturels, une origine et une langue commune, un héritage ancestral entendu comme "transmis de génération en génération ", un folklore et surtout des ancêtres (Lievre, 2013).

34 Notons par ailleurs que les catégories "Roms roumanisés " et " Roms traditionnels " prennent leur racine dans les écrits des voyageurs du début du XIXe siècle et c'est notamment sous l'influence des études sociologiques et ethnologiques qu'elles apparaissent en ces termes. Ce n'est qu'après 1989 que ces catégories vont petit à petit être naturalisées avec l'avènement des représentants roms roumains dont l'émergence repose sur une lutte symbolique entre ces deux types d'appartenance " être un Rom traditionnel vs roumanisé " (Lievre, 2013).

35 La dynamique de la " création des identités nationales " et du répertoire qui l'accompagne, le " système IKEA " (Thiesse, 1999 : 14) est un phénomène qui est loin d'être récent. II remonte à la fin du XIXe siècle (Hobsbawm, 1992 et 2012 ; Gellner, 1989). L'émergence du nationalisme ethnoculturel des Roms roumains s'étant surtout concrétisée depuis la transition communiste, nous pouvons observer une construction en formation et interroger son impact. 


\section{Conclusion}

Cet article a montré que l'usage de catégories désignées comme " ethniques ", et associées par ses locuteurs à un " degré " d'appartenance ethnique, traduit à l'inverse des processus de distinction sociale, et ce, pour tous les groupes roms présents à Montpellier. L'ethnicité étant une forme d'organisation sociale, ces catégories ne renseignent pas sur le fonctionnement de l'ethnicité, ni même sur leur degré de " tsiganité ": le cas des Popescu en est illustratif. Ceci étant, les logiques de distinction qui accompagnent l'usage de ces catégories peuvent éclairer et alimenter les normes et valeurs d'un groupe rom, c'est le cas des Cortorari. Par la lecture des caractéristiques posées comme marqueurs de différenciation, cet article montre que ces processus de distinction sociale viennent à poser comme saillantes dans la définition de soi des caractéristiques culturelles. Chacun de ces processus de différenciation sociale - dominés par la possession en capital culturel, économique et identitaire - alimente la définition de " nous ethniques ", dans ce que I'on peut nommer " un champ social des migrants roms ". Bien que révélateurs de processus de distinction sociale, c'est un rapport à la culture qui en émerge et parait omniprésent : réification d'une authenticité, d'une tradition, etc. La désignation des Ciurari comme les " ancêtres " des Tsiganes révèle un processus de domination sociale : en se différenciant socialement, les Popescu imposent en quelque sorte leur vision du monde social sur "l'authenticité "(Bourdieu, 1980). Ces observations ne sont pas sans faire écho aux catégories de pensée des représentants roms roumains sur la définition et l'existence du " peuple rom ". Exprimées comme catégories de la pratique, elles traduisent un processus d'intériorisation et révèlent la présence et la pénétration de l'idée de l'existence d'un " peuple tsigane aux origines communes " chez des Roms non politisés.

\section{Références bibliographiques}

Amselle Jean-Loup et M'Bokolo Elikia (1985) Au cœur de l'ethnie. Ethnies, tribalisme et État en Afrique, Paris, La Découverte, 238 p.

Auzias Claire (1995) Les Tsiganes ou Le destin sauvage des Roms de l'Est, Paris, Michalon, $130 \mathrm{p}$.

Avanza Martina et Laferté Gilles (2005) Dépasser la construction des identités ? Identification, image sociale, appartenance, Genèses, 61, pp. 134-152.

Babadzan Alain (2009) Le spectacle de la culture. Globalisation et traditionalismes en Océanie, Paris, L’Harmattan, 286 p.

Barth Fredrik (1995) Les groupes ethniques et leurs frontières, in Philippe Poutignat et Jocelyne Streiff-Fénart Éds., Théories de l'ethnicité, Paris, PUF, pp. 203-249.

Barth Fredrik (Ed.) (1969) Ethnic Groups and Boundaries:The Social Organization of Culture Difference, Bergen-Oslo, Universitets Forlaget.

Beissinger Margaret H. (2001) Occupation and Ethnicity: Constructing Identity among Professional Romani (Gypsy) Musicians in Romania, Slavic Review, $60(1)$, pp. 24-49. 
Benarrosh-Orsoni Norah (2015) Des maisonnées transnationales. Une migration rom dans ses routes, lieux et objets entre la Roumanie et la France, Thèse en ethnologie, Université Paris Ouest-Nanterre La Défense, 471 p.

Bourdieu Pierre (1998 [1979]) La Distinction. Critique sociale du jugement, Paris, Minuit, $670 \mathrm{p}$.

Bourdieu Pierre (1980) L'identité et la représentation : éléments pour une réflexion sur l'idée de région, Actes de la recherche en sciences sociales, 35 (1), pp. 63-72.

Brubaker Rogers (2001) Au-delà de l'« identité ", Actes de la recherche en sciences sociales, 139 (1), pp. 66-85.

Clavé-Mercier Alexandra (2015) Des États et des "Roms " : une anthropologie du sujet entre transnationalisme et politiques d'intégration de migrants bulgares en France, Thèse en ethnologie, Université de Bordeaux, 637 p.

Cossée Claire (2010) L'impossible neutralité des sciences sociales face aux entrepreneurs d'identité ethnique: "Gens du voyage ", "Rroms ", "Tsiganes " ou autres ethnonymes, Migrations Société, 22 (128), pp. 159-176.

Cousin Grégoire (2009) Rroms migrants. Tsiganes hier, Rroms migrants aujourd'hui ?, [en ligne] consulté le 02/08/2014. URL : http://www.espacestemps. net/articles/rroms-migrants/

Cuche Denys (2004 [3e éd.]) La notion de culture dans les sciences sociales, Paris, La Découverte, 123 p.

Fassin Éric, Fouteau Carine, Guichard Serge et Windels Aurélie (2014) Roms \& riverains. Une politique municipale de la race, Paris, La Fabrique, 227 p.

Gellner Ernest (1989 [éd. originale en anglais : 1983]) Nations et nationalismes, Paris, Payot, 208 p.

Géraud Marie-Odile, Leservoisier Olivier et Pottier Richard (2002) Les notions clés de l'ethnologie. Analyses et textes, Paris, Armand Colin, 332 p.

Gheorghe Nicolae (2012) Identitatea romani între victimizare şi emancipare. Nicolae Gheorghe în dialog cu lulius Rostaş, in István Horváth şi Lucian Nastasă Ed., Rom sau Tigan. Dilemele unui etnonim în spaţiul românesc, Cluj-Napoca, ISPMN, pp. 299-337.

Haşdeu lulia (2007) Bori, r(R)omni et Faraoance : Genre et ethnicité chez les Roms dans trois villages de Roumanie, Thèse en ethnologie, Université de Neuchâtel, sous la direction de Ellen Hertz, 306 p.

Hobsbawm Éric (1992) Nations et nationalisme depuis 1780, Paris, Gallimard, 255 p.

Hobsbawm Éric et Ranger Terence (Éds.) (2012 [1983]) L'invention de la tradition, Paris, Amsterdam, $370 \mathrm{p}$.

Liégeois Jean-Pierre (1976) Mutation Tsigane. La révolution bohémienne, Paris, Complexe, $227 \mathrm{p}$.

Lièvre Marion (2014) "Ceux-là sont peu soignés, peu débrouillards ». Ethnographie des Roms roumains migrants à Montpellier : entre enjeux moraux et appartenance sociale, Migrations Société, 26 (152), pp. 103-118.

Lièvre Marion (2013) Nationalisme ethnoculturel et rapport à la culture des Roms en Roumanie postcommuniste et multiculturaliste, Thèse en ethnologie, Université Paul-Valéry, Montpellier 3, $517 \mathrm{p}$. 
Legros Olivier et Vitale Tommaso (2011) Roms migrants en ville : pratiques et politiques en Italie et en France, Géocarrefour, 86 (1), [en ligne]. URL : https:// geocarrefour.revues.org/8220

Olivera Martin (2012) La tradition de l'intégration. Une ethnologie des Roms Gabori dans les années 2000, Paris, éditions Petra, 505 p.

Olivera Martin (2011) Roms en (bidon)villes. Quelle place pour les migrants précaires aujourd'hui ?, Éditions Rue d'Ulm, Paris, 84 p.

Olivera Martin (2010) Introduction aux formes et raisons de la diversité rom roumaine, Études tsiganes, "Roms de Roumanie, la diversité méconnue ", 38, pp. 10-44.

Piasere Leonardo (1985) Mare Roma, Catégories humaines et structure sociale, Paris, chez Paul-Henri Stahl, 274 p.

Poutignat Philippe et Streiff-Fénart Jocelyne (1995) Théories de l'ethnicité, suivi de Barth Fredrik, Les groupes ethniques et leurs frontières, Paris, PUF, 270 p.

Rădulescu Speranţa (2004) Taifasuri despre muzica ţigănească. Chats about gypsy music, Bucarest, Paideia, 247 p.

Rea Andrea et Tripier Maryse (2008) Sociologie de l'immigration, Paris, La Découverte, $128 \mathrm{p}$.

Reyniers Alain (1992) La roue et la pierre : contribution anthropo-historique à la connaissance de la production sociale et économique des Tsiganes, Thèse en sociologie, Université Paris V-Sorbonne, sous la direction de Louis-Vincent Thomas.

Tesăr Cătălina (2011) "Tigan bun traditional" în România, cerşetor de-etnicizat în străinătate. Politici ale reprezentării publice şi etica muncii la romii cortorari, in Stefánia Toma şi László Fosztó Eds., Spectrum. Cercetări sociale despre romi, Cluj Napoca, ISPMN, pp. 281-312.

Thiesse Anne-Marie (1999) La création des identités nationales, Paris, Seuil, 307 p.

Tonnies Ferdinand (1944) Communauté et société. Catégories fondamentales de la sociologie pure, Paris, PUF, $286 \mathrm{p}$.

Williams Patrick (1993) Nous, on n'en parle pas. Les vivants et les morts chez les Manouches, Paris, MSH, $108 \mathrm{p}$. 


\section{Marion Lièvre}

\section{Roms roumanisés, Ciurari, Ursari : ethnicité et appartenances sociales. Ethnographie des migrants roms roumains à Montpellier}

Comment devient-on Roms roumanisés, Ciurari, Ursari ou encore Bogdăneşteni? Sur la base d'une ethnographie des frontières et des marqueurs mobilisés par les différents groupes roms roumains présents à Montpellier, cet article interroge ce qui habituellement est désigné comme " culturel ". Appartenances ethniques, logiques de distinction sociale et processus de domination symbolique se conjuguent pour redessiner les contours des valeurs identitaires et culturelles de ces groupes roms et définir une " authenticité culturelle ".

\section{Romanized Roma, Ciurari, Ursari: Ethnicity and Social Belongings. Ethnography of Romanian Roma Migrants in Montpellier}

How does one become romanized Roma, Ciurari, Ursari or Bogdăneşteni? Based on an ethnography of boundaries and markers mobilized by the differents Romanian Roma groups established in Montpellier, this paper examines what is usually referred to as "cultural." Ethnics belongings, logic of social distinction, and processes of social domination combine to shape the contours of identity and cultural values of the Roma groups and define a "cultural authenticity".

\section{... Roma romanizados, Ciurari, Ursarí etnicidad y pertenencias sociales. Etnografía de los roma rumanos migrantes en Montpellier}

¿Cómo se convierte un individuo en roma romanizado, Ciurari, Ursari o Bogdăneşteni? Basándose en la etnografía de las fronteras y de los marcadores movilizados por los distintos grupos roma presentes en Montpellier, este artículo examina aquello que suele ser designado como "cultural». Pertenencias étnicas, lógicas de distinción social y procesos de dominación simbólica se conjugan para redibujar el contorno de los valores identitarios y culturales de esos grupos y definir una "autenticidad cultural». 